\title{
A health equity research agenda for India: results of a consultative exercise
}

\author{
T.K. Sundari Ravindran ${ }^{{ }^{*}}$ and Tanya Seshadri ${ }^{2}$ \\ From National Conference on Health Inequities in India: transformative research for action in Trivandrum, India (AMCCON 2018) \\ Trivandrum, India. 08-11 January 2018
}

\begin{abstract}
Background: This paper describes the process and outcome of a consultative exercise undertaken to develop a medium-term agenda for the next decade, and to identify a short list of immediate priorities for health equity research in India. This exercise was undertaken over 2014-2017 as part of 'Closing the Gap: Health Equity Research Initiative in India', implemented by the Achutha Menon Centre for Health Science Studies, at the Sree Chitra Tirunal Institute of Medical Sciences and Technology, Trivandrum, in south India.
\end{abstract}

Methods: We adopted a five-step process for the agenda- and priority-setting exercise. The first step, which lasted for approximately 1 year, consisted of a synthesis of evidence on health inequities in India produced during 2000-2014 and identification of gaps. In the second step, we shared the evidence gaps identified and engaged with diverse stakeholders to develop the research agenda through face-to-face and online consultations. In step three, we consolidated the research agenda and identified continuing gaps. Key informant consultations by phone or email with experts in the areas where gaps were identified constituted the fourth step. In the fifth and final step, we organised an expert group consultation to review the agenda and identify immediate research priorities through a consensus process. Overall, approximately 220 persons participated in the entire process, and consisted of persons from diverse disciplines and sectors.

Results: The research agenda and immediate priorities that emerged may be categorised into four themes, namely (1) descriptive research on the extent, nature and time trends in health inequities; (2) explanatory research on the pathways through which health inequities are created, and the political or policy environment that facilitates the process; (3) explanatory research that examines how health systems facilitate or mitigate inequities in healthcare; and (4) intervention research on initiatives that helped to mitigate health inequities, and examines the contributing factors.

Conclusion: The strength of this research agenda is that it was developed through a broad-based consultation with stakeholders representing diverse disciplines, sectors and constituencies. The use of this agenda will help generate evidence that will facilitate India moving closer to the Sustainable Development Goal of leaving no one behind.

Keywords: Health equity research, AMCCON 2018, Priority setting exercise, Health inequities in India

\footnotetext{
* Correspondence: ravindransundari@gmail.com

${ }^{1}$ Achutha Menon Centre for Health Science Studies, Sree Chitra Tirunal

Institute for Medical Sciences \& Technology, Trivandrum, Kerala, India

Full list of author information is available at the end of the article
}

(c) The Author(s). 2018 Open Access This article is distributed under the terms of the Creative Commons Attribution 4.0 International License (http://creativecommons.org/licenses/by/4.0/), which permits unrestricted use, distribution, and reproduction in any medium, provided you give appropriate credit to the original author(s) and the source, provide a link to the Creative Commons license, and indicate if changes were made. The Creative Commons Public Domain Dedication waiver (http://creativecommons.org/publicdomain/zero/1.0/) applies to the data made available in this article, unless otherwise stated. 


\section{Background}

Equality in general and health equity in particular are key themes in the Sustainable Development Goals (SDG) agenda for 2030 [1]. Equality in the SDGs refers to the right to not be discriminated against. In the context of health, health equity is the desired goal because inequalities in health may arise from genetic, biological or random factors. Health equity is defined as "unjust differences in health between persons of different social groups... [which] can be linked to disadvantages such as poverty, discrimination or lack of access to goods" [2]. India's National Health Policy 2017 also identified equity as a guiding principle [3]. Launching the new health policy in 2017, the Prime Minister of India stated: "the National Health Policy marks a historic moment in our endeavour to create a healthy India where everyone has access to quality healthcare" [4]. Programming for health equity calls for robust evidence on the concept, extent and nature of inequities in health, especially in view of scarce resources [5]. However, it is more important to generate evidence that unravels the factors and mechanisms that create, sustain and reinforce inequities. Nevertheless, the current evidence base on health inequities in India does not measure up to this task [6].

Disciplinary and sectoral boundaries fragment health equity research in India. Much of the academic research has been carried out by economists and public health researchers from a biomedical background, and each group has tended to examine issues from within its disciplinary perspective. A diverse group of civil society actors has also engaged in health equity research mainly to inform their field-based interventions and advocacy. There has been limited interaction between the academia and civil society actors working on health equity, with the subsequent absence of a community of health equity researchers to develop a common agenda [6]. One consequence of such fragmentation is the large research gap on important issues related to inequities in health in the country. Further, until recently, there have been no efforts to synthesise what is known and to develop a research agenda and priority areas of research on health inequities to inform national policy and programmatic action in India $[6,7]$.

In 2014, the Achutha Menon Centre for Health Science Studies at the Sree Chitra Tirunal Institute of Medical Sciences and Technology in south India, embarked on a project titled 'Closing the Gap: Health Equity Research Initiative in India'. The overall aim of the initiative was to "contribute to the advancement of a sound and actionable evidence-base on inequities in health in India with a view to influence government and civil society initiatives to prioritise the reduction of health inequities [8].” This 4-year project is supported by the International Development Research Centre, Canada.

A critical task in the project was to develop a medium-term agenda for the next decade and identify a short list of immediate priorities for health equity research in India. The research agenda is meant primarily for use by researchers from diverse disciplines and sectors, interested in or working on health equity research. At the same time, the agenda aims to inform research funding and research policy-making. This paper describes the exercise undertaken and its outcomes.

\section{Methods}

The methods adopted were informed by the following guiding principles, drawing on many years of experience in participatory research and action of key members of the Closing the Gap project, of which this activity was part.

- The agenda should focus on research that generates actionable evidence able to inform programming and policy-making to reduce health inequities, including increasing the visibility of health conditions and population groups about which/whom little is known

- The agenda-setting process should be consultative and iterative

- Multiple stakeholders should be involved in developing the agenda, including researchers as well as practitioners of diverse types such as policy-makers, programme managers, advocates and activists

- A conscious attempt should be made to represent the agendas of diverse marginalised groups

- The process should build on the already existing evidence base and draw on experiential knowledge

We undertook the agenda- and priority-setting exercise over a 4-year period (2014-2018) using a five-step process (Fig. 1).

\section{Step 1: Exploration and synthesis of evidence}

A detailed exercise was undertaken during 2014-2015 identifying public health research studies on health inequities in India, published during 2000-2014. The identification of studies was an iterative process, which included searches in major public health and social science databases and websites of organisations engaged with research on issues concerning the health of marginalised populations. We also consulted with researchers engaged in health equity research who pointed us to additional data sources. The search was limited to research available in English.

We developed a schema for the classification of health equity research across three dimensions, namely the nature of the research question, determinants of health inequities, and health conditions or outcomes studied (Table 1). This schema was arrived at after reviewing an initial set of studies identified for the synthesis. The schema was used to identify gaps in the evidence (Additional file 1). We found that most studies identified 


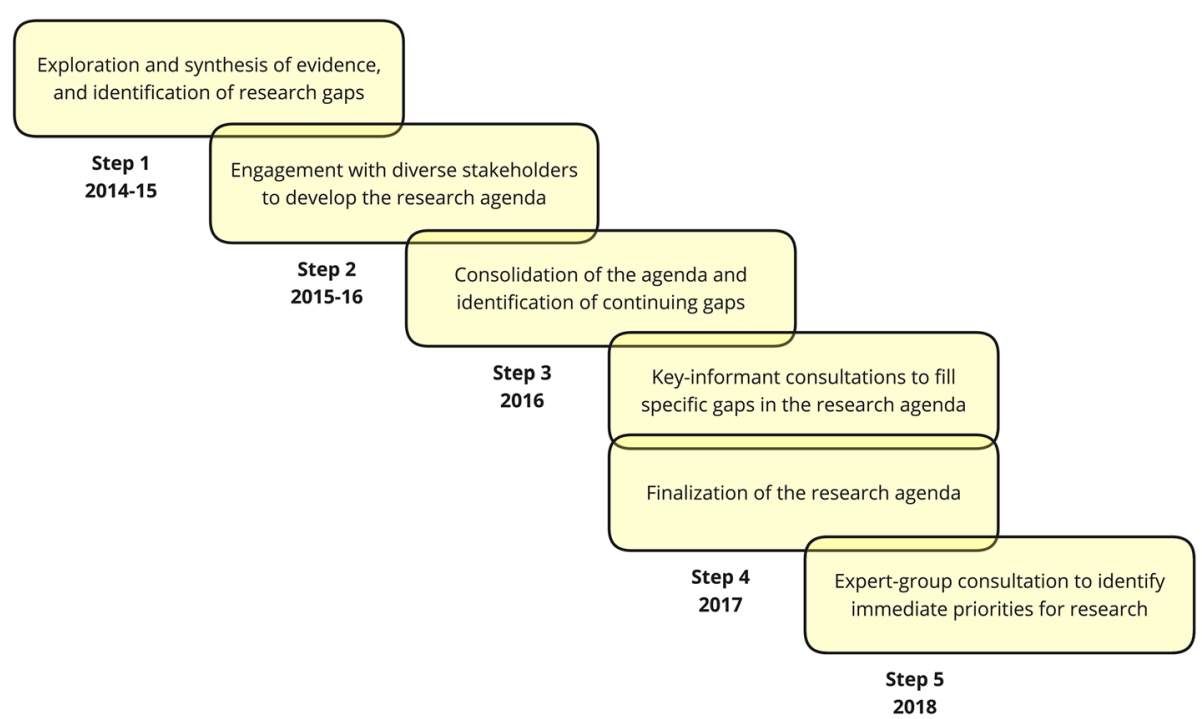

Fig. 1 The five-step process adopted for the agenda- and priority-setting exercise between 2014 and 2018

the existence of gaps in health outcomes associated with specific socioeconomic and demographic factors. Few studies went on to examine why and how these gaps came about. We also found that an overwhelming majority of the studies on health inequities were about maternal and child health. Of the five different axes or determinants of inequities examined, there was a dearth of studies on Dalit or Adivasi status, and evidence was especially scarce on health inequities experienced by vulnerable groups such as the elderly, persons living with physical and psycho-social disabilities, and persons of non-conforming sexual orientations and gender identities.
The schema (Table 1) also formed the basis for an evidence synthesis exercise (published elsewhere), and for soliciting themes for further research from multiple stakeholders [6]. We then used the same schema to organise the research agenda that emerged from the present exercise.

\section{Step 2: Engagement with diverse stakeholders to develop the research agenda}

This step lasted for approximately 1 year. Research gaps identified in Step 1 were presented to diverse stakeholders at two settings where research questions, areas and themes were gathered. The first was at a national

Table 1 Schema for organising the synthesis of research evidence and agenda

\begin{tabular}{|c|c|c|}
\hline Dimension & Themes & Research agenda and questions \\
\hline Dimension one & $\begin{array}{l}\text { Type of research } \\
\text { questions }\end{array}$ & $\begin{array}{l}\text { I. Descriptive research that answers the 'what', 'where' and 'when' questions on the extent, nature } \\
\text { and trends of health inequities } \\
\text { II. Explanatory research that answers the 'why' and 'how' questions on the pathways through } \\
\text { which health inequities are created, and the political/policy environment that facilitates the } \\
\text { process } \\
\text { III. Explanatory research that answers 'how' health systems create or facilitate inequities in } \\
\text { accessibility, affordability, acceptability, and quality } \\
\text { IV. Intervention research that answers the 'what works in addressing health inequities, } \\
\text { in which context, and why?' }\end{array}$ \\
\hline Dimension two & $\begin{array}{l}\text { Determinants } \\
\text { of health inequities }\end{array}$ & $\begin{array}{l}\text { I. Socioeconomic position } \\
\text { II. Caste/tribal status } \\
\text { III. Gender } \\
\text { IV. Other socially constructed vulnerable groups, e.g. people living with HIV/AIDS, migrants, elderly, } \\
\text { sexual minorities, sex workers, persons living with physical disabilities, persons living with mental } \\
\text { health conditions } \\
\text { V. Health systems }\end{array}$ \\
\hline Dimension three & Health conditions/outcomes & $\begin{array}{l}\text { I. Maternal health, wellbeing and nutrition } \\
\text { II. Child health, wellbeing and nutrition } \\
\text { III. Non-communicable diseases } \\
\text { IV. Communicable diseases } \\
\text { V. Violence and injuries } \\
\text { VI. Overall mortality, morbidity, nutrition }\end{array}$ \\
\hline
\end{tabular}


seminar on health inequities held in August 2015. The seminar brought together approximately 60 researchers, civil society actors and policy-makers with track records of engaging with health equity issues. In a session dedicated for this purpose, participants studied the research gaps emerging from the evidence synthesis and generated a list of broad areas, themes and research questions individually. Contributions from all the participants were collected and compiled. The second was an online consultation through the health inequities web portal (http://www.healthinequity.com) to consult diverse constituencies. This web-portal of the Health Equity Research Initiative in India features regular updates on project relevant activities and newsletters. Once again, the research gaps were shared, and contributions to the research agenda were solicited by email. The web portal has a membership of approximately 450 researchers and activists from diverse sectors.

\section{Step 3: Consolidation of the agenda and the identification of continuing gaps}

The questions/themes generated through stakeholder engagement were consolidated and organised, once again, according to the schema described in Table 1. The consolidation led to the identification of continuing gaps in research questions related to health inequities. In particular, these were related to specific groups about whom little evidence is available related to health inequities, specifically persons living with physical and psychosocial disabilities and mental health problems, the elderly, persons of non-conforming gender identities or sexual orientations (Lesbian, Gay, Bisexual, Transgender, Queer and Intersex communities), and migrant populations.

\section{Step 4: Key-informant consultations to fill specific gaps in} the research agenda; finalisation of the research agenda

In 2017, for each of the four areas for which research areas had not been identified through the earlier steps, specific persons with expertise in the identified areas were contacted by email or phone, briefed about the exercise and requested to contribute to the research agenda. We contacted at least 5-6 persons for each of the four areas, and research questions/themes were elicited in consultation with them.

At the end of Step 4, a list of 231 research questions/ themes was prepared. Next, the research themes and questions were consolidated. The project team clubbed together similar questions, eliminated repetitions, distilled questions and themes for clarity, and organised all the questions according to dimension one of the initial schema, namely types of research questions.

Approximately 200 persons participated in steps 2 to 4 . Of these, participants in the national seminar (Step 2) had the opportunity to have a face-to-face presentation on the evidence gaps, discuss with each other and hand-in their ideas on what should go into the research agenda. This group of approximately 60 people consisted predominantly of senior public health experts and senior civil society actors working on health equity, with many combining research and activism, and 10 policy-makers from the central and state governments. Approximately 120 people who participated in the online consultations (Step 2) were predominantly young and intermediate-career public health and social science researchers. The key informant interviews (Step 3 ) included approximately 20 researchers and civil society actors from key under-represented constituencies.

\section{Step 5: Expert group consultation to identify immediate priorities for research in health equity}

From the medium-term (10-year) research agenda that we had generated, we wanted to identify a subset of immediate priorities for research. The basis for prioritising was one or more of the following: (1) potential for action; (2) potential for filling a crucial evidence gap; and (3) potential for expanding conceptual understanding of health inequities and pathways as well as processes contributing to it.

This last step consisted of an expert group consultation held at the National Conference on Health Inequities in India, organised by Achutha Menon Centre for Health Science Studies on January 8-11, 2018, at Trivandrum, India. A closed group consultation with experts was held as part of the Conference, in which 20 experts reviewed the research agenda. The experts were from diverse backgrounds and often wore several hats simultaneously. For example, many were civil society actors cum researchers, or mainly researchers who also engaged in advocacy and activism. In terms of disciplines, there were health systems researchers, epidemiologists, social scientists and anthropologists. In terms of areas of focus, there were persons working on issues related to caste- and ethnicity-based discrimination, gender and rights of persons living with mental health problems, disabilities and of persons with non-conforming gender identities and sexual orientation. We chose to prioritise through consensus, first within the small groups and then within the larger group of all 20 experts.

The expert group broke-out into four subgroups of five each. Each group reflected on one of the four categories of research questions and came up with priority areas or questions for immediate engagement. The priority areas and questions were usually drawn from the larger research agenda but were sometimes an addition to it. Each group discussed and arrived at a list of priorities, and then presented their top priorities in the large group. A list of immediate (next 2-3 years) priorities were identified in each research category through discussion and consensus in the group. 
In the next section, we present the research agenda and immediate research priorities for each of the four categories of research questions.

\section{Results}

\section{Research agenda and priorities identified}

The medium-term research agenda and priority questions that emerged from the 4-year consultative process were organised into the following four groups, based on the nature of the research question (Table 1).

1) Descriptive research that answers the 'what', 'where' and 'when' questions on the extent and nature and time trends of health inequities

2) Explanatory research that answers the 'why' and 'how' questions on the pathways through which health inequities are created and the political or policy environment that facilitates the process

3) Explanatory research that answers 'how' health systems facilitate or mitigate inequities in accessibility, affordability, acceptability and quality of healthcare

4) Intervention research that answers the question 'what works in addressing health inequities, in which context, and why?

In this section, we present the medium-term (10-year) research agenda organised according to the above categories and, within each category, we also present areas identified as immediate priorities.

\section{Descriptive research that answers the 'what', 'where' and 'when' questions on the extent and nature and time trends of health inequities}

We have termed 'descriptive' studies that describe the existence of health inequities, note the nature of the gaps across locations and over time. Many descriptive studies identify economic position, caste, tribal status or sex as correlates of differentials in health outcomes. Some of them also track changes (or lack thereof) in health outcomes over time. Many social groups experiencing vulnerabilities and marginalisation have not been the subject of descriptive studies on health inequities.

Descriptive studies are important to establish that specific population groups experience health inequities, and to motivate further studies into the reasons underlying the observed inequities. In the Indian context, there are several vulnerable population groups about whom such information is not available. The research agenda identified 12 groups who have been least represented in the evidence on health inequities, about whom it is important to initiate descriptive studies on health inequities. Studies are needed which describe the health situation of these groups, locating it in the context of population averages or comparing it with the health outcomes of groups known to enjoy greater power and privileges. For each of these groups, studies are needed on health behaviours as well as health outcomes. Table 2 presents the research agenda for descriptive studies on the least studied population groups.

The research agenda for descriptive studies on health inequities has a second part, which pertains to groups whose experience of health inequities is well established. Apart from some small-scale studies, there are a large number of studies analysing data from National Family Health Surveys, from Sample Registration Surveys and National Sample Surveys. For such population groups, the need is to go beyond the analysis of the next round of national surveys and to look at more complex themes even within descriptive studies.

The research agenda for more complex descriptive studies include the following:

1) Looking at within-group health inequities in vulnerable groups (e.g. within the group of Dalits or Adivasis, of women and men, of low-income groups)

2) Examining the consequences to health inequities of intersections of multiple vulnerabilities (e.g. elderly

Table 2 A research agenda for descriptive studies on least studied population groups

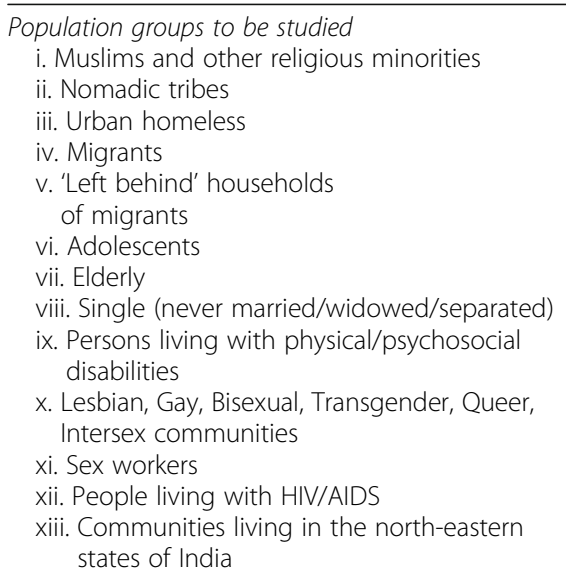

Population groups to be studied

ii. Nomadic tribes

iii. Urban homeless

. 'Left behind' households

of migrants

vi. Adolescents

viii. Single (never married/widowed/separated)

x. Persons living with physical/psychosocial

Intersex communities

Sex workers

xiii. Communities living in the north-eastern states of India

\section{Health outcomes}

i. Overall health needs of specific populations

ii. Specific health conditions about which there is limited information (e.g. cervical cancer); specific health conditions in specific population groups (e.g. tuberculosis in elderly or internal migrants)

iii. Ignored health needs of specific populations, e.g. beyond sexual and reproductive health for adolescents,

beyond HIV for people living with HIV/AIDS

iv. Nutritional status

i. Quality of life, perceived psychological and physical wellbeing

Health behaviours

i. Health literacy/awareness of healthy behaviours and symptoms of health problems

ii. Care-seeking behaviour (from whom, after how many days, for which conditions)

iii. Access and utilisation, unmet need for healthcare/treatment compliance/treatment completion and barriers to these

iv. Experience with healthcare providers/in healthcare facilities 
by class and gender, adolescents by rural/urban location and age, migrants by rural-to-urban or urban-to-urban migration)

3) Changes over time and differences across geographic locations of health inequities (e.g. changes over time in caste-based or gender-based health inequities)

4) Comparing relative position in the social gradient of different marginalised groups (e.g. Dalits compared to Adivasis compared to Muslims)

The set of immediate priorities for descriptive research studies called for a focus on persons living with disabilities, on the different communities in the north-east region of India, on within-group stratification among Dalit and Adivasi groups, and on health conditions beyond maternal and child health. A more detailed list is presented in Table 3.

\section{Explanatory research that answers the 'why' and 'how' questions, on the pathways through which health inequities are created, and the political/policy environment that facilitates the process}

For some population groups, including Dalits, Adivasis, low-income groups, women in specific settings, residents of rural areas, urban slums or poorly performing districts or states, existing research has focused on the nature and extent of the disadvantage they experience but has seldom gone deeper so as to understand the reasons for the disadvantage. Studies within these groups need to shift gear and move towards explanatory studies. Concerning each of these population groups, the research agenda for explanatory studies on social processes leading to health inequities calls for a focus on questions that explain how health inequities have come about or are sustained. The two broad strands of questions are (1) what are the social processes that translate a specific social location into disadvantages in terms of access to resources and power, and through these, to poor health? (e.g. social exclusion, discrimination, stigma which may be the pathway through which Adivasi households are deprived of access to health resources), and (2) what are the macro-level socioeconomic and political determinants creating conditions that widen or narrow social stratification, contributing to health inequities? (e.g. cuts in public spending on the social sector, informalisation of labour, corporate control over healthcare).

The outcome variables to be examined are similar to those listed for descriptive studies, namely relevant behaviours and health outcomes, including morbidity, mortality and well-being, and access to and utilisation of healthcare and the quality of care received. As in the case of descriptive research studies, so also with explanatory research studies, the research agenda calls for exploring multiple axes of vulnerabilities. Explanatory research explores whether the mechanisms and processes underlying inequities differ for groups experiencing multiple disadvantages and provides valuable insight. However, to study mechanisms and underlying processes, research also needs to focus on building theoretical, conceptual and methodological tools. The key research priorities identified are summarised in Table 3.

\section{Explanatory research that answers 'how' health systems create, reinforce or mitigate inequities}

The third category of research questions regards the role of the health system in facilitating or mitigating health inequities. The purpose of health systems is to ensure a basic level of healthcare for all. However, health systems are also social institutions, embedded in the fabric of the society of which they are a part [9]. As a consequence, unless explicit and conscious policy measures are adopted, health systems are most likely to reflect the hierarchies and power relations of the context within which they are located. Thus, while health systems have the potential to uphold values of equitable and universal access, and respect the human rights of all its users, they could also reinforce and perpetuate health inequities and be blind to discrimination against vulnerable populations. For all these reasons, health systems are a crucial domain of inquiry when researching the mechanisms underlying health inequities.

Explanatory studies are needed on the role of health systems in caste, gender and socioeconomic status-based inequities in health in the poor health of various vulnerable population groups, similar to those identified for research questions in category two above. With respect to each of these population groups, the research agenda for explanatory studies on health inequities calls for a focus on questions that explain how health inequities have come about and are sustained. The two broad strands of questions are (1) how do the structure of the health system (e.g. public/private mix, distribution of services across levels of care, the extent of decentralisation, financing), the design of service delivery, the distribution of human and financial resources, and the processes of decision-making within the health system affect health inequities? (e.g. the requirement of residence permits may exclude migrant workers from accessing services, the lack of a woman doctor may discourage women from accessing gynaecological services of a sensitive nature); and (2) how do factors at the global and national levels influence the structure and functioning of the health system (e.g. government policies on the privatisation of healthcare, World Trade Organisation's intervention to alter the pharmaceutical scenario, employment opportunities abroad for nurses).

The outcome variables of interest are accessibility, acceptability, affordability and quality of healthcare services 


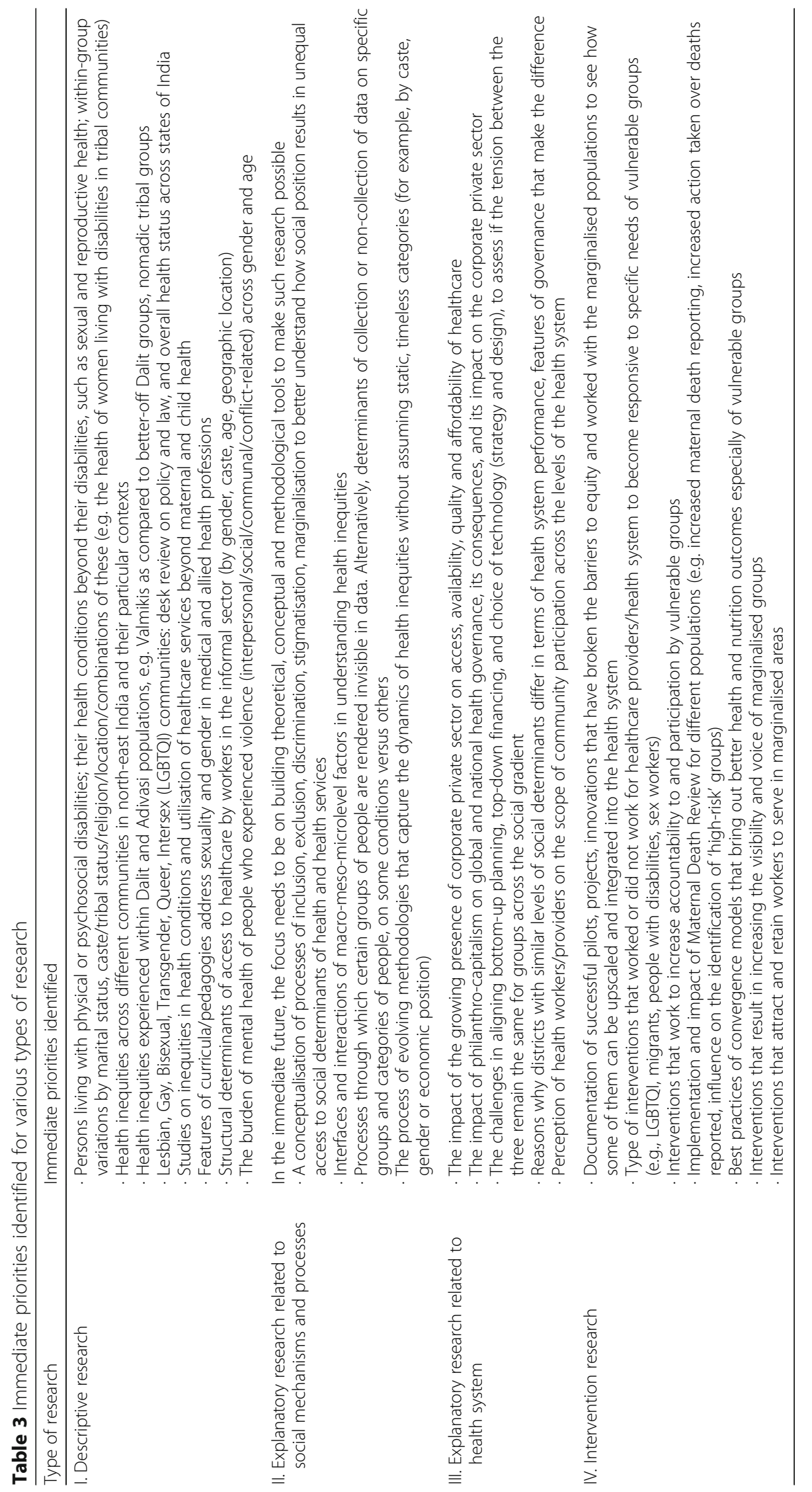


and inequities, and their socioeconomic consequences in these across population groups. The key research priorities identified are summarised in Table 3.

\section{Intervention research that answers the question 'what works in addressing health inequities, in which context, and why?'}

Finally, moving from asking 'what,' 'why' and 'how' questions for various population groups and existing health inequities, it is crucial for research to also support action in terms of developing or evaluating interventions that aim to address the health inequities identified. We classified the different interventions that deal with health inequities into the following categories: (1) interventions aimed at improving health outcomes (for instance, those aimed at reducing infant and maternal mortality); (2) interventions that target specific population groups (like children or elderly) or locations (like high priority districts); (3) interventions that attempt to improve awareness or influence health-related behaviours; and (4) interventions that aim to improve access to social determinants of health, thereby improving their health outcome (like access to better housing or nutritious food).

The research agenda on interventions research called for a focus on both descriptive and explanatory studies. There is a dearth of descriptive studies that explain in detail the health inequities addressed by the intervention, actors involved, strategies adopted, the theory of change of the intervention, challenges of implementation, and the outcomes in terms of success or failure in reducing the health inequities targeted.

The research agenda for explanatory studies on health equity interventions include studies that examine the reasons why some interventions succeed while others do not. Among the range of factors are the context, actors, strategies, implementation process and governance, not only at the local level but also at the macro level. The key research priorities identified for intervention research are summarised in Table 3.

The 4-year-long exercise described herein had many limitations. The research gaps identified were based on a synthesis exercise limited to studies published in the English language, which may have resulted in the exclusion of salient evidence published in other Indian languages. Further, the consultative process with stakeholders in a national seminar and through online calls for participation did not yield the expected results. Despite receiving more than 150 responses, some of the population groups on whom there was a major evidence gap were poorly represented in the research agenda, and we had to introduce an additional step of consultation with key informants to address this. Further, the short list of immediate priorities includes not only what emerged through the broad-based consultations but also specific concerns of a small group of experts. Finally, our consultations included civil society actors representing the interests and concerns of many vulnerable groups, and not the groups themselves, which would have called for resources that were not within the scope of our project.

Despite these limitations, the process adopted was consultative and inclusive, drew stakeholders from many disciplines including early and mid-career researchers and senior experts. The medium-term research agenda and immediate priorities for health equity research in India include a comprehensive range of research questions, ranging from the descriptive to the analytical, and encompassing intervention research. While much of the research agenda consists of empirical research questions, there was also an emphasis on theory-building based on findings on the ground. This was based on the finding from Step 1 of this exercise that public health research in health inequities seldom based itself on theoretical knowledge either from within the discipline or from other disciplines.

\section{Conclusion}

The strength of this research agenda is that it was developed through a broad-based consultation with stakeholders representing diverse disciplines, sectors and constituencies. It aims to motivate and inspire public health researchers to ask the right questions, answers to which will help unravel the mechanisms and pathways contributing to health inequities, and to identify interventions that will contribute to redressing inequities. We also hope that those who sponsor and fund public health research will draw on this research agenda to identify key funding priorities on health inequities research, to generate evidence that will help India move closer to the SDG goal of leaving no one behind.

\section{Additional file}

Additional file 1: Research gaps identified through the mapping and synthesis exercise. (PDF $33 \mathrm{~kb}$ )

\section{Acknowledgements \\ We would like to thank everyone who contributed to developing the research agenda and especially the group of experts who identified the immediate research priorities. We would like to thank the International Development Research Centre, Canada, for funding this research project. We would also like to thank colleagues and students at the Achutha Menon Centre for Health Science Studies at Sree Chitra Tirunal Institute of Medical Sciences \& Technology in Trivandrum, Kerala, for their support in implementing this research.}

Funding

The cost of publication is sponsored by the Closing the Gap: Health Equity Research Initiative in India project, funded by the International Development Research Centre.

\section{About this supplement}

This article has been published as part of Health Research Policy and Systems Volume 16 Supplement 1, 2018: Health inequities in India: transformative research 
for action. Highlights from a conference. The full contents of the supplement are available online at https://health-policy-systems.biomedcentral.com/articles/ supplements/volume-16-supplement-1.

\section{Authors' contributions}

TKS is the principal investigator for the Closing the Gap: Health Equity Research Initiative in India, and conceived, planned and conducted the exercises under the project. TS participated in planning and conducting part of the exercises. TKS \& TS both contributed towards writing of this paper. Both authors read and approved the final draft for publication.

\section{Ethics approval and consent to participate}

Not applicable.

\section{Consent for publication}

Not applicable.

\section{Competing interests}

The authors declare that they have no competing interests.

\section{Publisher's Note}

Springer Nature remains neutral with regard to jurisdictional claims in published maps and institutional affiliations.

\section{Author details}

${ }^{1}$ Achutha Menon Centre for Health Science Studies, Sree Chitra Tirunal Institute for Medical Sciences \& Technology, Trivandrum, Kerala, India.

${ }^{2}$ Independent researcher, Bengaluru, India.

Published: 9 October 2018

\section{References}

1. United Nations. Transforming our World: The 2030 Agenda for Sustainable Development. 2015. https:/sustainabledevelopment.un.org/content/documents/ 21252030\%20Agenda\%20for\%20Sustainable\%20Development\%20web.pdf. Accessed 27 Jul 2018.

2. World Health Organization. Handbook on Health Inequality Monitoring with a Special Focus on Low- and Middle-income Countries. Geneva: WHO; 2013.

3. Press Information Bureau. National Health Policy, 2017 Approved by Cabinet Focus on Preventive and Promotive Health Care and Universal Access to Good Quality Health Care Services. 2017. http://pib.nic.in/newsite/ PrintRelease.aspx?relid=159376. Accessed $27 \mathrm{Jul} 2018$.

4. Modi N. 2017. Twitter 16 March. https:/twitter.com/narendramodi/status/ 842369652957237251. Accessed 27 Jul 2018

5. Braveman P. Health disparities and health equity: concepts and measurement. Annu Rev Public Health. 2006;27(1):167-94.

6. Ravindran T, Gaitonde R. Health Inequities in India: A Synthesis of Recent Evidence. 1st ed. Singapore: Springer; 2018.

7. Bhan N, Rao K, Kachwaha S. Health inequalities research in India: a review of trends and themes in the literature since the 1990s. Int J Equity Health. 2016;15(1):166

8. Closing the Gap: Health Equity Research Initiative in India', project proposal submitted to IDRC, Canada, March 2014

9. World Health Organization. Everybody's Business: Strengthening Health Systems to Improve Health Outcomes. Geneva: WHO; 2007.

Ready to submit your research? Choose BMC and benefit from:

- fast, convenient online submission

- thorough peer review by experienced researchers in your field

- rapid publication on acceptance

- support for research data, including large and complex data types

- gold Open Access which fosters wider collaboration and increased citations

- maximum visibility for your research: over $100 \mathrm{M}$ website views per year

At $\mathrm{BMC}$, research is always in progress.

Learn more biomedcentral.com/submissions 